\title{
The fibrotic tumor stroma
}

\author{
Mitsuo Yamauchi,, Thomas H. Barker, ${ }^{2}$ Don L. Gibbons,, ${ }^{3,4}$ and Jonathan M. Kurie ${ }^{3}$ \\ ${ }^{1}$ Oral and Craniofacial Health Sciences, School of Dentistry, University of North Carolina at Chapel Hill, Chapel Hill, North Carolina, USA. ${ }^{2}$ Department of Biomedical Engineering, School of Engineering and \\ Applied Sciences and School of Medicine, University of Virginia, Charlottesville, Virginia, USA. ${ }^{3}$ Department of Thoracic/Head and Neck Medical Oncology and ${ }^{4}$ Department of Molecular and Cellular Oncology, \\ University of Texas MD Anderson Cancer Center, Houston, Texas, USA.
}

\begin{abstract}
Intratumoral fibrosis results from the deposition of a cross-linked collagen matrix by cancer-associated fibroblasts (CAFs). This type of fibrosis has been shown to exert mechanical forces and create a biochemical milieu that, together, shape intratumoral immunity and influence tumor cell metastatic behavior. In this Review, we present recent evidence that CAFs and tumor cells are regulated by provisional matrix molecules, that metastasis results from a change in the type of stromal collagen cross-link, and that fibrosis and inflammation perpetuate each other through proteolytic and chemotactic mediators released into the tumor stroma. We also discuss aspects of the emerging biology that have potential therapeutic value.
\end{abstract}

\section{Introduction}

Over the past two centuries, numerous clinical and pathological observations have established a clear relationship between chronic inflammation, fibrosis, and cancer (1). Skin fibrosis associated with recessive dystrophic epidermolysis bullosa leads to highly metastatic skin carcinomas (2). Progressive lung scarring associated with idiopathic pulmonary fibrosis is a risk factor for lung cancer development (3). Moreover, human pancreatic adenocarcinomas can be highly fibrotic, and experimental evidence in animals supports an etiologic role for fibrosis in pancreatic cancer progression $(4,5)$. Thus, fibrosis can precede or follow cancer development and may participate in multiple stages of tumorigenesis and metastasis. In this Review, we present recent findings on the composition of the fibrotic tumor stroma and how its mechanical and biochemical effects influence diverse intratumoral processes that determine the metastatic fate of tumor cells. We speculate on therapeutic opportunities, pose questions, and discuss future challenges in this rapidly expanding field.

\section{Provisional matrix deposition in cancer: priming for fibrosis}

The concept that tumors represent "wounds that do not heal" was put forward over 30 years ago (6). Subsequently, R.A. Clark coined the term "provisional matrix" to describe factors that appeared coincident with epidermal cell migration during skin wound healing $(7,8)$. In the context of fibrosis and cancer, the provisional matrix can provide initial instructions to resident and invading immune and inflammatory cells and stromal cell populations (i.e., perivascular cells, resident stem/progenitor cells, and quiescent fibroblasts) that activate them toward a pro-wound repair state (9) and, in certain cases, provide cues that stimulate epithelial-tomesenchymal transition (EMT) and endothelial-to-mesenchymal transition (refs. 10, 11, and Figure 1). Although reparative during

Conflict of interest: D.L. Gibbons received laboratory research funding from Janssen Research and Development.

Reference information: / Clin Invest. 2018;128(1):16-25.

https://doi.org/10.1172/JCI93554. normal wound healing processes, the provisional matrix loses reparative capacity in the tumor stroma owing to physical and posttranslational modifications that occur during myofibroblast remodeling. Although deep proteomic sequencing of tumor matrices has revealed hundreds of proteins that are aberrantly expressed and/or modified (12), here we will address how several of the most abundant matrix components (fibrin, fibronectin, and collagen) can regulate mechanical properties of the tumor stroma.

The provisional matrix, composed primarily of fibrin and fibronectin, plays a critical role in angiogenesis, a process by which new blood vessels grow from existing vessels that is critical in healing wounds and is strongly associated with tumor progression. Rapid, disorganized angiogenic responses driven by tumor oxygen requirements result in persistent activation of the coagulation cascade and deposition of fibrin-rich early provisional matrix. In 2013, Iacoviello et al. found a strong correlation between plasminogen activator inhibitor-1 (PAI-1) and elevated risk of colorectal and breast cancer, suggesting that this critical regulator of fibrin persistence is a potential risk factor (13). Links between the coagulation cascade, fibrin persistence, and fibrosis are equally strong, suggesting a common pathway between cancer and fibrosis. For instance, mice overexpressing PAI-1 display enhanced fibrosis, whereas PAI-1 knockdown protects against fibrosis in various models, including bleomycin-induced lung fibrosis (14-16). In urokinase- and plasmin-deficient mice, fibrin persistence in injured muscle is linked to poor regeneration and replacement fibrosis $(17,18)$. While PAI-1 and other enzymes regulating fibrin degradation, such as urokinase, are perturbed in these mice, so too are enzymes impacting the formation of fibrin. Procoagulant factors such as fibrinogen and factors VII and X are elevated in patients with intra-alveolar fibrosis (19). Thrombin, in particular, can directly stimulate fibroblast proliferation (20), extracellular matrix (ECM) production (21), and differentiation into myofibroblasts expressing $\alpha$-smooth muscle actin ( $\alpha$ SMA), all of which are characteristic of the tumor-associated fibrotic reaction known as desmoplasia (22). Fibrin persistence also greatly impacts the inflammatory milieu of the tumor stromal microenvironment because of fibrin's ability to bind macrophage- 1 antigen (Mac-1) 
Early provisional ECM

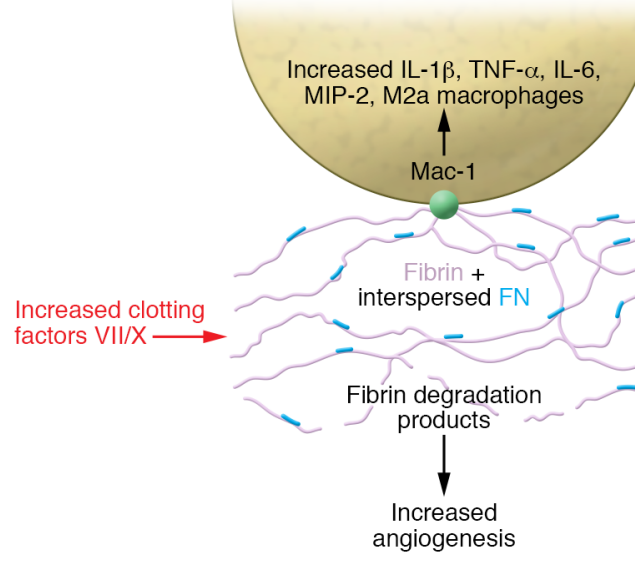

Late provisional ECM

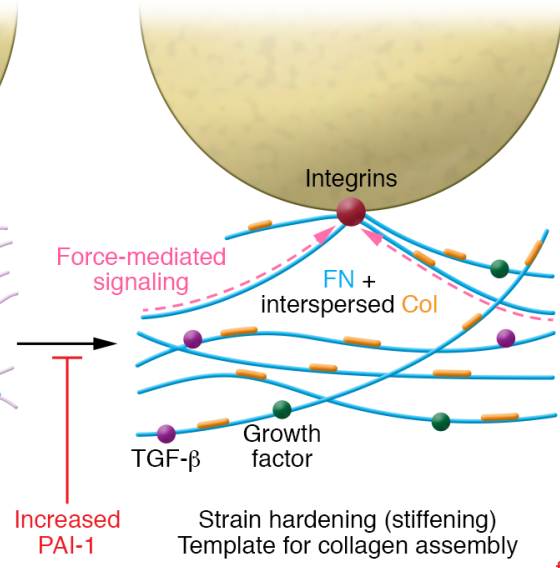

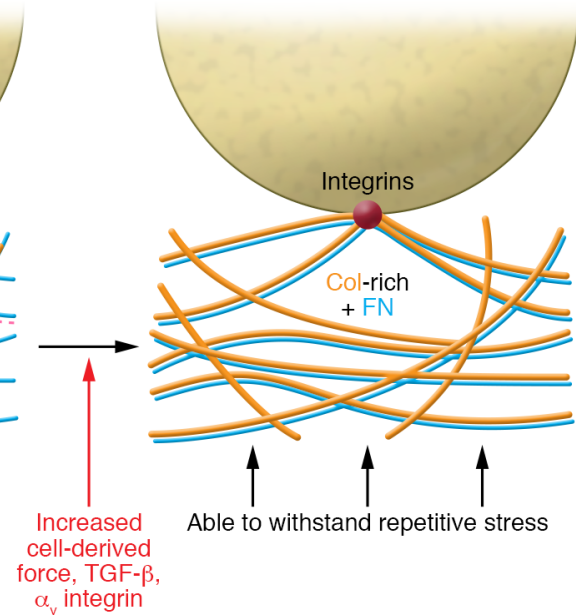

Figure 1. The provisional matrix primes for fibrosis. Early provisional matrix is primarily composed of fibrin. Fibrin interacts with macrophage-1 antigen (Mac-1) to upregulate proinflammatory cytokines that signal to resident and invading immune cells as well as stromal cell populations. The degradation products of fibrin play a key role in angiogenesis, leading to persistent activation of the coagulation cascade and promoting fibrin persistence. Increases in fibronectin indicate a shift to late provisional extracellular matrix (ECM) and serve as scaffolding for growth factors and mechanical signaling. At this stage, an increasingly stiff ECM serves as a template for collagen (Col) deposition. Finally, mature ECM is characterized by increased density of type I collagen as well as the ability to resist degradation and repetitive mechanical stress. FN, fibronectin; MIP-2, macrophage inhibitory protein-2; PAI-1, plasminogen activator inhibitor-1.

on classically activated macrophages. Fibrin engagement of Mac-1 leads to upregulation of the proinflammatory cytokines IL-1 $\beta$, TNF- $\alpha$, IL-6, and macrophage inhibitory protein-2 (MIP-2) (23). In models of dystrophic muscle (which present a strong fibrotic phenotype), persistent fibrin leads to increasing presence of alternatively activated macrophages (M2a) (21), which have been shown to promote fibrosis in several model systems $(24,25)$.

Fibronectin (FN), another key component of the provisional matrix, serves as a signal scaffolding and growth factor-depot protein through its multiple and spatially coordinated binding sites for receptors, growth factors, and other ECM proteins (26). Plasma FN is initially deposited into the interstitial space during fibrin polymerization, where it binds and is cross-linked to the fibrin-rich early provisional matrix. FN, like fibrin, can bind directly to invading inflammatory cells, vascular cells, and fibroblasts, where it triggers cell adhesion and invasion into the early provisional matrix. Invading fibroblasts secrete and assemble an elaborate FN-rich transitional ECM (the so-called late provisional matrix) that lays the foundation of tissue progression. FN-rich ECM generated by cancer-associated fibroblasts (CAFs) has been shown to be sufficient to drive desmoplastic differentiation of normal fibroblasts and can facilitate metastasis of cancer cells (27). FN can signal directly to cells through its ability to engage a wide range of cellular integrins (26), bind a plethora of growth factors (28), and spatially arrange growth factor receptors and integrins to facilitate synergistic signaling (29-31). Importantly, FN is a depot for TGF- $\beta$ latent complex, where it has been shown to be physically coupled to the fibrillary FN matrix. This physical coupling ensures that TGF- $\beta$ is readily available to cells despite transcriptional regulation of this key fibrosis-associated growth factor. The physical/structural nature of fibrillary FN matrix is also a key signaling characteris- tic of FN (e.g., topotaxis, also known as topographical guidance of invasion). FN is also highly flexible as a result of labile type III repeats, which enable molecular extension and exposure of cryptic binding sites, leading to mechanical regulation of FN's biochemical function. For example, activation of FN-bound latent TGF- $\beta$ has been demonstrated by simply stretching the matrix $(32,33)$. Furthermore, force-mediated molecular extension of FN can alter its growth factor binding profile. We have shown that molecular extension of FN's integrin-binding domain occurs in a model of lung fibrosis and angiogenesis (34) and that such conformational changes drive altered integrin binding that can lead to fibrosisassociated cellular phenotypes such as EMT $(10,35)$. These findings suggest that molecular extension represents a fundamental mechanism by which $\mathrm{FN}$ mechanosensitivity regulates fibrosis formation and resolution (or lack thereof). At an even more fundamental level, both the topography (fiber architecture) and the biophysical properties (i.e., stiffness, strain-hardening) of FN-rich ECMs can impact cell behaviors, leading to profibrotic $(36,37)$ and cancer phenotypes (38-40). Indeed, in other recent studies it has been shown that presentation of a partially unfolded variant of the FN integrin-binding domain induces tumor-like vasculature within an engineered biomaterial (41). The dynamic and integrative biochemical and biophysical signaling nature of FN is of critical importance in the context of mechanically active myofibroblasts, whose phenotype is partially described by their contractile properties and their association with a stiffening microenvironment (in the context of both tumor stroma and tissue fibrosis), which reciprocally activates fibroblasts to become myofibroblasts $(36,37,42)$. Finally, one must not forget that $\mathrm{FN}$ also serves as the functional scaffold for nascent collagen deposition by fibroblasts (43), a critical step in tumor growth and metastasis. FN is truly the gateway matrix. 
A
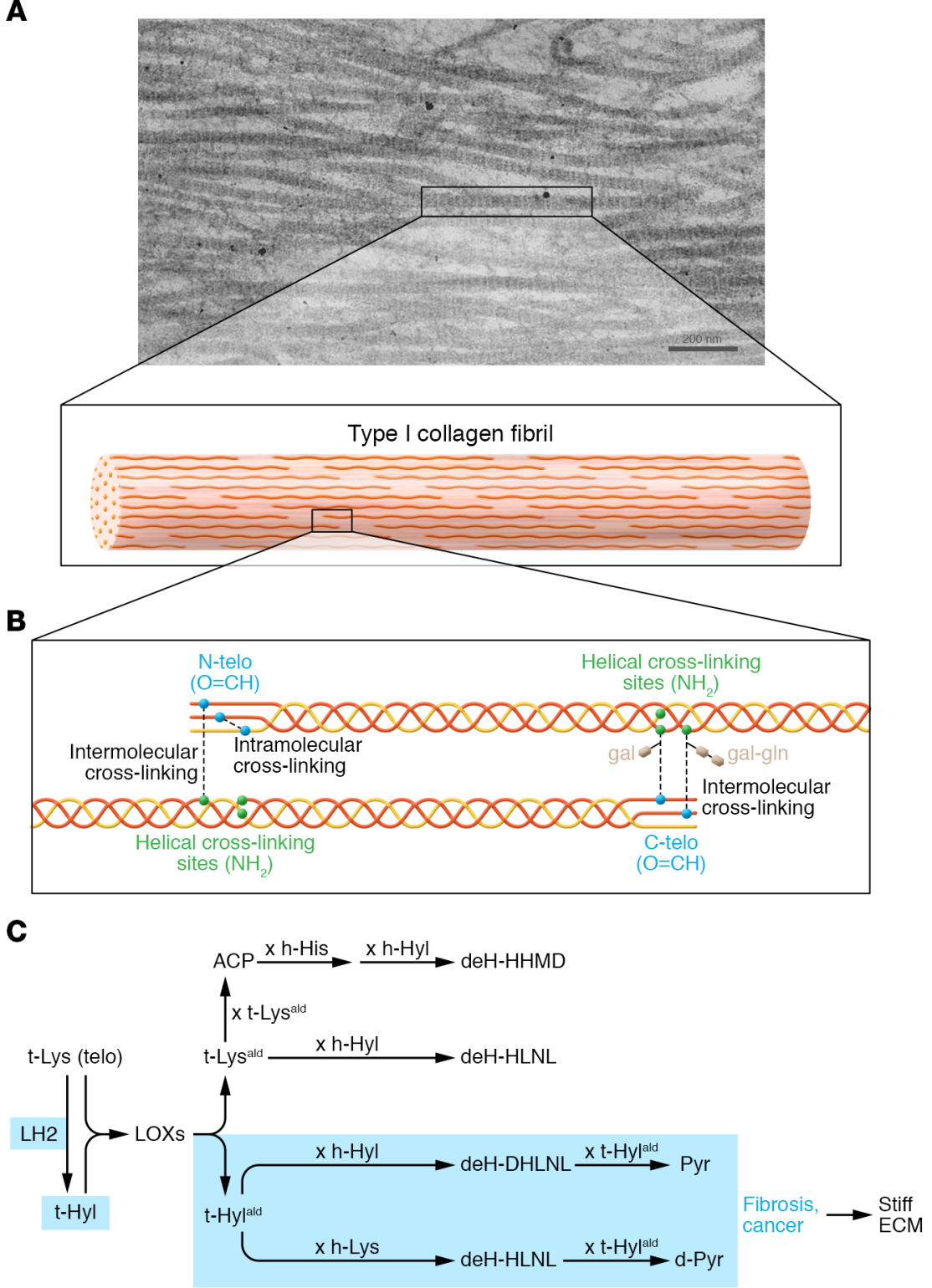

Figure 2. Collagen cross-linking in fibrosis and cancer. (A) Transmission electron microscopic image of tumor tissue showing dense type I collagen fibrils (reprinted with permission from the Journal of Biological Chemistry, ref. 126). Rectangle indicates a single fibril. Below, a sketch represents type I collagen molecules (wavy lines) packed in a fibril in parallel and staggered with respect to one another by approximately $67 \mathrm{~nm}$. (B) Cross-linking sites of adjacent collagen molecules. Initial cross-links are indicated by dashed lines. Two telopeptidyl lysine aldehydes $(\mathrm{O}=\mathrm{CH})$ can cross-link within the same molecule (intramolecular cross-link). Telopeptidyl lysine- and hydroxylysine-aldehyde $(\mathrm{O}=\mathrm{CH})$ can condense with $\varepsilon$-amino groups $\left(\mathrm{NH}_{2}\right)$ of juxtaposed helical lysine or hydroxylysine residues to form intermolecular crosslinks. Helical cross-linking hydroxylysine residues near the $\mathrm{N}$-terminus are one of the major glycosylation sites (galactose or galactose-glucose indicated in tan) in type I collagen, contributing to glycosylated cross-linking. (C) Major cross-linking pathways characterized in fibrotic and cancer tissues (for comprehensive cross-linking pathways, see ref. 65). Lysyl oxidases (LOXs) initiate cross-linking by converting lysine or hydroxylysine residues in the telopeptides to aldehyde (t-Lys ${ }^{\text {ald }}$ and $t-H y{ }^{\text {lad }}$, respectively). Then the aldehyde condenses with another t-Lys ${ }^{\text {ald }}$ in the same molecule or juxtaposed helical Lys (h-Lys) or $\mathrm{Hyl}(\mathrm{h}-\mathrm{Hyl}$ ) on a neighboring molecule. These divalent cross-links can then mature into tri- and tetravalent cross-links. All cross-links are intermolecular crosslinks except an aldol condensation product (ACP). In fibrosis and cancer, the pathway is driven toward LH2-mediated Hylald-derived pathway, as indicated by blue boxes (115). d, deoxy; deH, dehydro; DHLNL, dihydroxylysinonorleucine; h, helical; HHMD, histidinohydroxymerodesmosine; HLNL, hydroxylysinonorleucine; LH2, lysyl hydroxylase-2; Pyr, pyridinoline; t, telopeptidyl.

\section{Fibroblasts arbitrate the desmoplastic reaction and cancer-associated fibrosis}

If wound healing and fibrosis represent the progression of ECM deposition, degradation, and remodeling toward a more permanent collagen-rich ECM, then the fibroblast must be considered the arbiter of that progression. The host's response to a growing tumor involves heterotypic interaction and paracrine signaling between cancer cells, vasculature, immune cells, and fibroblasts in the desmoplastic reaction within the tumor microenvironment (44-46). The fibroblasts within this reaction, which are collectively termed CAFs, have diverse origins and phenotypes (47). Studies on genetically engineered mouse models of cancer have revealed remarkable CAF heterogeneity (48). Several groups have attempted to define the heterogeneity of CAFs based on functional outcomes, which seems appropriate given the lack of definitive, fibroblastspecific markers. In one classification scheme, CAFs are clearly distinguished from quiescent, tissue-resident fibroblasts and even wound-associated activated fibroblasts. Tissue-resident fibroblasts are in a resting, nonproliferative, low metabolic state and, in some cases, are thought to serve as progenitor or stem cells (49). Wound-associated activated fibroblasts are characterized by loss of certain fibroblast markers (e.g., fibroblast-specific protein-1), acquisition of muscle-like markers (e.g., aSMA), increased ECM synthesis, and enhanced ECM remodeling (5055). Key distinctions between the wound-associated activated fibroblast and CAFs or fibrosis-associated fibroblasts are the sensitivity of wound-associated activated fibroblasts to apoptosis and their ability to facilitate wound resolution through clearance by natural killer cells, a process known as nemosis, and/or dedifferentiation back to a resting state $(52,56)$. Furthermore, evidence suggests that CAFs, but not normal fibroblasts, support metastatic lesions (57), implying greater differences than simply a resistance to apoptosis. The exact mechanism driving this transition into a CAF or fibrotic fibroblast remains unknown but is likely an adaptive response to the chronic wound healing reaction and accompanying inflammatory milieu associated with the 


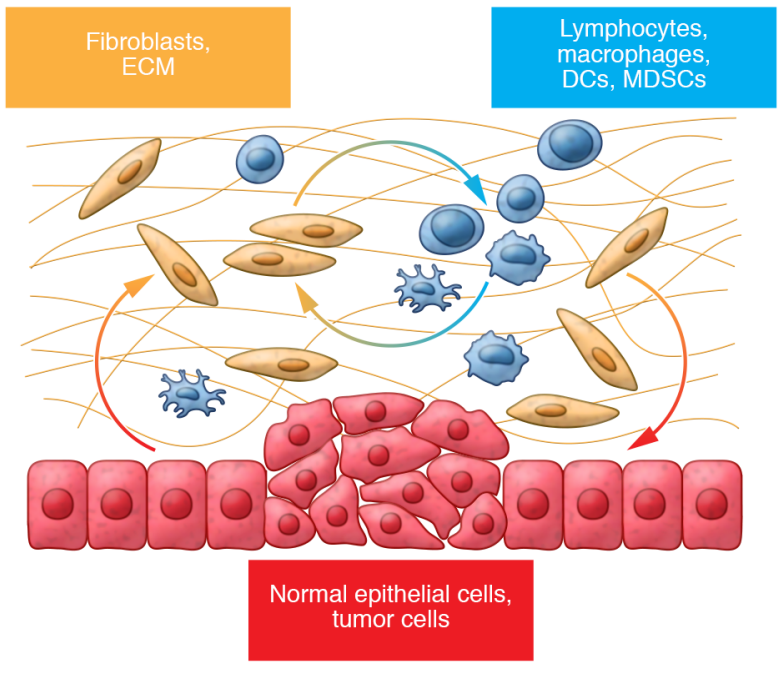

Figure 3. Interconnections between cells in the tumor microenvironment regulate ECM composition and immune surveillance. ECM, extracellular matrix; MDSC, myeloid-derived suppressor cell.

growing tumor. CAFs are recruited to the tumor stroma primarily through the actions of growth factors, such as TGF- $\beta$, plateletderived growth factor, and fibroblast growth factor-2, which are also regulators of tissue repair responses. TGF- $\beta$ induces CAF proliferation, expansion, and myofibroblastic differentiation as well as EMT. This important chemokine has also demonstrated a potential role in tumor suppression through the induction of apoptosis, which tumor cells evade through mutation of SMAD4 in the TGF- $\beta$ axis (58).

The importance of the tumor stroma in regulating cancer biology is undisputed, even if the exact role it plays remains incompletely understood. CAFs and their stroma appear to have context-dependent functions, and data support both tumorrestrictive and -supportive roles. In the early stages of neoplasia, the inflammatory milieu may skew resident and invading fibroblasts toward a proinflammatory and protumorigenic phenotype. Cytokines such as IL- $1 \beta$ and TNF- $\alpha$ activate NF- $\kappa$ B, thereby stimulating fibroblasts to secrete protumorigenic factors. Whether these initial inflammatory signals cause more permanent adaptations in the fibroblasts through epigenetic regulation of miR-21 (59) and other mediators is unclear but could explain the persistence of CAF phenotypes. Fibroblasts are also inherently sensitive to both the biophysical and the biochemical nature of the structural ECM; increased stiffness activates key mechanosensitive transcription factors such as YAP/TAZ (60) and myocardin-related transcription factor (36) and drives profibrotic/prostromal remodeling phenotypes through their transcriptional regulation of $\mathrm{FN}$, collagen, periostin, $\alpha \mathrm{SMA}$, and other ECM proteins. CAFs themselves appear to mechanically prime their microenvironment by driving the formation of collagen cross-links that may stabilize their phenotype and influence the invasive properties of resident tumor cells (61). The Cukierman group recently described an interplay between an FN-rich stromal ECM and normal fibroblasts that leads to CAF development and activation, which occurs via a TGF- $\beta$-independent regulation of $\alpha_{v} \beta_{5}$ integrin-associated redistribution of $\alpha_{5} \beta_{1}$ integrin into endosomes (62). This work identified emergent CAF subtypes that have opposing effects on clinical outcome, highlighting the importance of CAF stratification systems for future clinical efforts to target the tumor stroma. In a recent review, Kalluri proposed a more generalized classification of CAFs into tumor-restraining (F1), tumor-promoting (F2), secretory (F3), and ECM-remodeling (F4) (47). Whether such functional classifications appropriately stratify the diverse heterogeneity of CAFs and their broad impacts on ECM remodeling remains unclear.

\section{Fibrillar type I collagen and intermolecular cross-linking}

The tumor stroma transitions from a provisional matrix to a dense fibrosis by accumulating fibrillar collagens, among which type I collagen is the predominant component. Fibrillar collagen accumulation and stiffening can result from increased collagen synthesis that is driven by profibrotic cytokines, particularly TGF- $\beta 1$, impaired collagen degradation due to an imbalance between matrix metalloproteinases (MMPs) and their inhibitors, and the formation of specific covalent intermolecular cross-links that render collagen fibers resistant to MMP-mediated degradation. The importance of TGF- $\beta 1$, MMPs, and MMP inhibitors has been extensively reviewed elsewhere $(63,64)$. In the following section, we will review the basic collagen cross-linking chemistry and how it is altered in fibrosis and cancer.

Type I collagen, a heterotrimeric molecule composed of two $\alpha 1$ chains and one $\alpha 2$ chain, consists of three structural domains: amino-terminal nonhelical telopeptide, central triple-helical (helical), and carboxy-terminal nonhelical telopeptide domains (65). The central helical domain of each chain contains more than 300 repeats of X-Y-Gly sequence representing more than $95 \%$ of the polypeptide. In the collagen fibril, molecules are packed in parallel, longitudinally staggered with respect to one another by approximately $67 \mathrm{~nm}$, and stabilized by covalent intermolecular cross-linking (Figure 2, A-C), which is the final step of collagen biosynthesis (for collagen biosynthesis, see refs. 66, 67). Covalent intermolecular cross-linking occurs between telopeptide and helical domains on adjacent collagen molecules through interactions between key lysine (Lys), 5-hydroxylysine (Hyl), and histidine (His) residues (Figure 2, B and C). Cross-linking is a multistep process that is regulated at the levels of cross-link initiation, crosslink type determination, and cross-link maturation. At each level of regulation, Lys posttranslational modifications play critical roles and, with the exception of the final condensation reactions, are enzymatically controlled.

Helical and telopeptidyl Lys on collagen can be hydroxylated to form Hyl residues, and specific helical Hyls are further modified by O-linked glycosylation to produce galactosyl-Hyl (G-Hyl) or glucosyl-galactosyl-Hyl (GG-Hyl). Lys hydroxylation is catalyzed by lysyl hydroxylases 1-3 (LH1-LH3) encoded by procollagenlysine, 2-oxyglutarate 5-dioxygenase genes (PLOD1-PLOD3) $(68,69)$, and, like prolyl hydroxylation, the reaction requires $\mathrm{Fe}^{2+}, 2$-oxoglutarate, $\mathrm{O}_{2}$, and ascorbate (70). LH2 is the only LH family member that can hydroxylate Lys residues within the telopeptide sequences -X-Lys-Ala-, -X-Lys-Ser-, and X-Lys-Gly. LH1 hydroxylates primarily Lys residues within the helical domain of collagen (e.g., -X-Lys-Gly-), and LH3 functions principally as a glycosyltransferase by transferring a glucose unit 
to G-Hyl to form GG-Hyl $(71,72)$. The major glycosylation in type I collagen occurs at the helical cross-linking sites near the $\mathrm{N}$-terminus, and it may regulate cross-link maturation (72-75). Recent studies showed that LHs are regulated by a number of ER-resident chaperones and foldases (76-82). Defects in these $\mathrm{LH}$-associated proteins result in abnormal collagen cross-links that lead to bone and connective tissue disorders $(81,83,84)$. These new findings clearly indicate that Lys modifications are tightly regulated at multiple levels and are critically important in connective tissue development.

Collagen cross-linking is initiated in the extracellular space by the action of lysyl oxidase (LOX) and LOX-like (LOXL) family members (for a review, see refs. 85-87), which catalyze the oxidative deamination of Lys and Hyl residues in the telopeptides, generating reactive aldehydes ( $\mathrm{Lys}^{\text {ald }}$ and $\mathrm{Hyl}^{\text {ald }}$, respectively). These aldehydes then undergo a series of condensation reactions with vicinal Lys ${ }^{\text {ald }}$, Lys, Hyl, and His residues to form tissuespecific covalent intra/intermolecular cross-links (ref. 65 and Figure 2, B and C). Lys ${ }^{\text {ald }}$-derived collagen cross-links (LCCs), for instance, are particularly abundant in soft connective tissues such as skin and cornea (88-91). Hylald-derived collagen crosslinks (HLCCs) are abundant in large load-bearing skeletal tissues such as bones and cartilage and are generally more stable than LCCs $(89,92)$. Cross-linking pattern is also altered under pathological conditions. For detailed chemistry and biology of collagen cross-linking, see several review articles $(88,92)$.

\section{How collagen cross-linking is altered in cancer}

LOX and LOXL2 levels are elevated in numerous cancer types (93-99). Secretion of LOX by tumor cells $(96,100)$ initiates collagen cross-linking and thereby stiffens tumor stroma, creating mechanical forces that trigger integrin-mediated formation of focal adhesions that initiate tumor cell invasion (101). Thus, LOX may drive metastasis, in part, by increasing the amount of collagen cross-links in tumor stroma. More recent studies have opened a new frontier of cancer research by showing that, in addition to the quantity of cross-links, the "type" of cross-links can also influence tissue mechanics within tumor stroma. This principle is based on several decades of research on fibrotic diseases. For example, in fibrotic diseases of the skin such as lipodermatosclerosis and keloid, the predominant types of cross-links switch from LCCs to HLCCs as a result of overhydroxylation of the telopeptidyl Lys residues $(102,103)$. Similarly, HLCCs are the predominant type of cross-link in fibrotic diseases of the lung $(104,105)$ and liver (106-108). The collagen cross-link switch in fibrosis results from increased expression of LH2 in fibroblasts $(109,110)$. In the initial studies on LH2 in cancer, several groups reported that high LH2 levels promote metastasis and are correlated with shorter durations of survival (111-116). Several of these studies showed that LH2 stabilizes and organizes the collagen matrix in tumor stroma. By comprehensively analyzing the collagen cross-links in cancer, we showed that LH2 increases HLCCs at the expense of LCCs, leading to a change in the predominant type, but not necessarily the quantity, of collagen cross-links (115). LH2 and LOX are coordinately upregulated in response to hypoxia and are direct targets of hypoxia-inducible factor- $1 \alpha$, suggesting that hypoxia regulates both the type and quantity of collagen cross-links as part of an integrated profibrotic response (112-122). The finding that cancer recapitulates the fibrosis-associated switch toward a highHLCC, low-LCC state provides a potential biochemical basis for the prometastatic effect of LH2 (Figure 2C). Providing provocative evidence that the collagen maturation process can adversely impact clinical outcome, Keely and colleagues identified a paradigm of type I collagen maturation that is associated with tumor growth in genetically engineered mouse models of human breast cancer and applied those metrics to a human breast cancer cohort, which showed that the presence of type I collagen bundles that orient perpendicularly to the tumor boundary is correlated with a shorter duration of survival (123).

Until recently, LH2 was considered to be a dedicated resident of the ER, where it hydroxylates Lys residues on procollagen $\alpha$ chains before the formation of triple helix $(66,124)$. In that context, the finding that tumor cell-derived LH2 increases HLCC formation in tumor stroma is counterintuitive, because tumor cell-derived LH2 should have limited access to collagen originating from CAFs, the primary source of fibrillar collagen in tumor stroma (125). Two recent findings by our group offer a potential explanation to this apparent paradox. First, in addition to residing on the ER, LH2 is secreted by tumor cells and can modify collagen in the extracellular space (126). Second, LH2 is expressed not only in tumor cells but also in CAFs, which can switch the tumor stroma toward a high-HLCC, low-LCC state (61).

\section{Fibrosis and intratumoral immune surveillance}

Infiltration of innate and adaptive immune cells is a prominent feature of the activated tumor microenvironment. CAFs and tumor cells deposit matrix proteins and matrix-modifying enzymes that impact immune cell recruitment and function; conversely, activated immune cells modify the microenvironment in ways that affect matrix composition and structure, creating an interlocking cycle of immune cell recruitment and ECM production (Figure 3). The last few years have witnessed a revolution in cancer therapy based on immuno-oncology (127). A better understanding of the way in which cancer-associated fibrosis regulates immune cell function will be critical to developing improved immunotherapies.

In normal tissues, epithelial cells create an antiinflammatory milieu (128) and produce basement membrane structures that differentially regulate immune cell trafficking, activation, and function (129, 130). This model of tissue-based immune cell homeostasis depends on matrix composition, which includes fibrillar collagens (mainly type I, but also types II, III, V, and XI), nonfibrillar collagens, glycoproteins, and a mix of small and large proteoglycans $(130,131)$, to create architectural features that alter the ability of leukocytes to traverse the tissue by their use of non-integrin-dependent, non-proteasedependent mechanisms of migration $(132,133)$. As opposed to the more variable mesh-like architectures of interstitial and provisional matrix, basement membranes have a very dense protein network that functions to separate tissue compartments $(134,135)$. These structural and biochemical compositional differences are selective for immune cell migration. For example, immune cells adhere to the laminin 511 isoform, which is found in a patchy distribution on endothelial basement membranes and provides an inhibitory signal to cell migration, driving cells toward regions containing high laminin 411, an isoform that supports cell migration $(130,136,137)$. 
Intratumoral fibrosis and inflammation perpetuate each other through enzymatic and chemotactic mediators (138). For example, CAFs regulate the recruitment and activation of immune cells (139-142) through multiple mechanisms, including CXCL12 secretion $(143,144)$, establishment of a competitive metabolic microenvironment (145), and skewing of inflammation toward Th2 and Th17 responses (146-149). Proteolytic degradation of ECM components by MMPs and other enzymes exposes damage-associated molecular patterns (DAMPs) that trigger an orchestrated inflammatory response through pattern recognition receptors on immune cells $(150,151)$. This system is analogous to the DAMPs that mediate innate immune response to infection, the best characterized of which are the small leucinerich proteoglycans decorin and biglycan that drive inflammation via activation of TLR2/4 signaling on macrophages, dendritic cells, and T cells. Beyond their biochemical actions, ECM molecules have architectural properties that govern immune cell function in tumors; for example, the spacing and orientation of collagen in tumors can have a "trapping" effect on T cells as described above for normal tissues (132, 133, 152-154). However, effector T cells and other immune cell types have been detected in areas of dense fibrosis in pancreatic cancer (155), arguing against a dominant role for mechanical impedance of immune cell infiltration by collagen. Conversely, immune cells that are recruited to early sites of neoplasia release cytokines that reprogram normal fibroblasts into CAFs (142) and MMPs that remodel collagen and activate the release of profibrotic cytokines (156). On the basis of evidence from wound repair models showing that the early fibrotic reaction driven by M1 macrophages and Th2 $\mathrm{CD}^{+} \mathrm{T}$ cells is eventually suppressed by a later influx of M2 macrophages and Th1/Th17 CD4 ${ }^{+} \mathrm{T}$ cells (156), intratumoral fibrosis is likely to be dynamic owing to maturation of intratumoral immunity during tumor progression.

\section{Therapeutic implications}

The fibrotic tumor stroma is an emerging target in cancer therapeutics. Antifibrosis drugs studied thus far in preclinical and clinical trials were recently reviewed (157). Two drugs recently approved for the treatment of idiopathic pulmonary fibrosis are of interest. Although its precise target is unclear, pirfenidone reduces tissue fibrosis, inhibits fibroblast proliferation, decreases the expression of profibrotic mediators such as TGF- $\beta$, reduces collagen synthesis (158-160), and suppresses the proinflammatory mediators IL-1 $\beta$ and TNF- $\alpha$ (161). Pirfenadone has demonstrated efficacy in animal models of fibrosis (162). Nintedanib is a multireceptor tyrosine kinase inhibitor that primarily targets receptors for vascular endothelial growth factor, fibroblast growth factors, and platelet-derived growth factors, which are important drivers of fibrosis in the lung $(163,164)$. Combination chemotherapy strategies that include nintedanib have demonstrated a survival benefit in cancer (165). Other antifibrosis drugs are in preclinical and early clinical trials. For example, $\alpha_{v}$ integrin inhibitors have demonstrated activity in fibrosis models (166).

With increasing tumor stiffness, intratumoral blood and lymphatic vessels compress and eventually close, which causes interstitial fluid to accumulate, generate increased pressure, and reduce efficient drug transport across blood vessel walls (167).
These findings provide a compelling rationale to alleviating tumor stromal stiffness as a means of improving drug delivery. On the basis of evidence that depleting CAFs or collagen can reduce tumor stiffness (167), preclinical studies were performed with a sonic hedgehog inhibitor to target CAFs or with antifibrotic agents (e.g., pirfenidone, losartan, tranilast, or hyaluronidase), which improved tumor perfusion, drug delivery, and treatment efficacy (167-169). On the basis of these findings, a phase II clinical trial with losartan and FOLFIRINOX is under way in pancreatic cancer (ClinicalTrials.gov, NCT01821729).

Efforts are also under way to target collagen-modifying enzymes. Neutralizing antibodies against LOX or LOXL2 have efficacy in preclinical cancer models $(93,121,170-176)$, but clinical trials that combined anti-LOXL2 antibodies with chemotherapy failed to show increased efficacy $(177,178)$. These approaches may have failed because of early metastatic escape, which has been reported in pancreatic ductal adenocarcinoma (179). Minoxidil has been used to inhibit LH2 in preclinical models (180), but its mode of action is unclear. LH2 might also be targeted indirectly with tacrolimus, which inhibits FKBP65, a peptidyl prolyl isomerase that enhances LH2 enzymatic activity $(79,80)$. Developing selective inhibitors of collagen-modifying enzymes will require insight into the structural properties of their active sites, but crystal structures of these enzymes have not been reported.

Genetic and pharmacologic strategies to deplete CAFs in genetically engineered mouse models of cancer have yielded disparate outcomes. In pancreatic cancer models, disruption of sonic hedgehog/smoothened-dependent signaling in fibroblasts prolonged survival and chemotherapy responsiveness in one study (181) and accelerated cancer progression in another (182). Genetic depletion of proliferating $\alpha \mathrm{SMA}^{+}$myofibroblasts in pancreatic cancer models substantially decreased myofibroblasts and fibrosis (183), and the CAF-depleted tumors displayed a more aggressive phenotype and alterations in regulatory $\mathrm{T}$ cells that increased responsiveness to anti-CTLA4 therapy, raising the possibility that CAF-targeting strategies may have both beneficial and detrimental effects. In an alternative therapeutic concept, CAFs can be reprogrammed to a normal fibroblast-like state that suppresses tumorigenesis $(4,138,184)$. Supporting this concept, agonists of the vitamin $\mathrm{D}$ receptor, a master regulator of pancreatic and hepatic stellate cells (184), inhibit stromal fibroblasts in pancreatic cancer and combine with gemcitabine to dramatically reduce tumor size. Similar findings were observed when all-trans retinoic acid was used to reverse pancreatic stellate cell activation, which also caused profound increases in cytotoxic T cell infiltration into tumors (144). Macrophage activation by CD40 agonists led to depletion of the fibrotic matrix and active tumor cell killing (185), suggesting that immune strategies may have the beneficial secondary effect of reversing tumor-associated fibrosis.

\section{Summary}

Recent findings show that fibrosis plays a central role in regulating the hallmark features of cancer. However, large gaps in our understanding remain and must be addressed in order to develop a comprehensive model that can be used to selectively target CAF populations and ECM molecules for the purpose of arresting tumorigenesis and metastasis, the key driver of cancer-related mortality. 


\section{Acknowledgments}

This work was supported by the NIH through R01 CA105155 (to JMK and MY), R01 HL127283 (to THB), R01 HL132585 (to THB), and R21 AR060978 (to MY); and by Cancer Prevention Research Institute of Texas Multi-investigator Research Awards RP120713 (to JMK and DLG) and RP160652 (to JMK and DLG). JMK holds the Elza A. and Ina S. Freeman Endowed Professorship in Lung Cancer. DLG is an
R. Lee Clark Fellow of the University of Texas MD Anderson Cancer Center supported by the Jeane F. Shelby Scholarship Fund.

Address correspondence to: Jonathan M. Kurie, MD Anderson Cancer Center, Box 432, Department of Thoracic/Head and Neck Medical Oncology, 1515 Holcombe Boulevard, Houston, Texas 77030, USA. Phone: 713.745.6747; E-mail: jkurie@mdanderson.org.
1. Brücher BL, Jamall IS. Epistemology of the origin of cancer: a new paradigm. BMC Cancer. 2014;14:331.

2. Guerra L, Odorisio T, Zambruno G, Castiglia D. Stromal microenvironment in type VII collagendeficient skin: the ground for squamous cell carcinoma development. Matrix Biol. 2017;63:1-10.

3. Karampitsakos $\mathrm{T}$, et al. Lung cancer in patients with idiopathic pulmonary fibrosis. Pulm Phar macol Ther. 2017;45:1-10.

4. Neesse A, Algül H, Tuveson DA, Gress TM. Stromal biology and therapy in pancreatic cancer: a changing paradigm. Gut. 2015;64(9):1476-1484.

5. Laklai $\mathrm{H}$, et al. Genotype tunes pancreatic ductal adenocarcinoma tissue tension to induce matricellular fibrosis and tumor progression. Nat Med. 2016;22(5):497-505.

6. Dvorak HF. Tumors: wounds that do not heal. Similarities between tumor stroma generation and wound healing. N Engl JMed. 1986;315(26):1650-1659.

7. Clark RA, Lanigan JM, DellaPelle P, Manseau E, Dvorak HF, Colvin RB. Fibronectin and fibrin provide a provisional matrix for epidermal cell migration during wound reepithelialization. J Invest Dermatol. 1982;79(5):264-269.

8. Barker TH, Engler AJ. The provisional matrix setting the stage for tissue repair outcomes. Matrix Biol. 2017;60-61:1-4.

9. Comoglio PM, Trusolino L. Cancer: the matrix is now in control. Nat Med. 2005;11(11):1156-1159.

10. Brown AC, Fiore VF, Sulchek TA, Barker TH. Physical and chemical microenvironmental cues orthogonally control the degree and duration of fibrosis-associated epithelial-to-mesenchymal transitions. J Pathol. 2013;229(1):25-35.

11. Piera-Velazquez S, Li Z, Jimenez SA. Role of endothelial-mesenchymal transition (EndoMT) in the pathogenesis of fibrotic disorders. Am J Pathol. 2011;179(3):1074-1080.

12. Naba A, Clauser KR, Ding H, Whittaker CA, Carr SA, Hynes RO. The extracellular matrix: tools and insights for the "omics" era. Matrix Biol. 2016;49:10-24.

13. Iacoviello L, et al. Type 1 plasminogen activator inhibitor as a common risk factor for cancer and ischaemic vascular disease: the EPICOR study. BMJOpen. 2013;3(11):e003725.

14. Eitzman DT, et al. Bleomycin-induced pulmonary fibrosis in transgenic mice that either lack or overexpress the murine plasminogen activator inhibitor-1 gene. J Clin Invest. 1996;97(1):232-237.

15. de Giorgio-Miller A, Bottoms S, Laurent G, Carmeliet $\mathrm{P}$, Herrick S. Fibrin-induced skin fibrosis in mice deficient in tissue plasminogen activator. Am J Pathol. 2005;167(3):721-732.

16. Loskutoff DJ, Quigley JP. PAI-1, fibrosis, and the elusive provisional fibrin matrix. JClin Invest.
2000;106(12):1441-1443.

17. Lluís F, et al. Urokinase-dependent plasminogen activation is required for efficient skeletal muscle regeneration in vivo. Blood. 2001;97(6):1703-1711.

18. Suelves M, et al. The plasminogen activation system in skeletal muscle regeneration: antagonistic roles of urokinase-type plasminogen activator (uPA) and its inhibitor (PAI-1). Front Biosci. 2005;10:2978-2985.

19. Peyrol S, Cordier JF, Grimaud JA. Intra-alveolar fibrosis of idiopathic bronchiolitis obliteransorganizing pneumonia. Cell-matrix patterns. Am J Pathol. 1990;137(1):155-170.

20. Chen LB, Buchanan JM. Mitogenic activity of blood components. I. Thrombin and prothrombin. Proc Natl Acad Sci U S A. 1975;72(1):131-135.

21. Chambers RC, Dabbagh K, McAnulty RJ, Gray AJ, Blanc-Brude OP, Laurent GJ. Thrombin stimulates fibroblast procollagen production via proteolytic activation of protease-activated receptor 1. Biochem J. 1998;333(pt 1):121-127.

22. Bogatkevich GS, Tourkina E, Silver RM, LudwickaBradley A. Thrombin differentiates normal lung fibroblasts to a myofibroblast phenotype via the proteolytically activated receptor- 1 and a protein kinase C-dependent pathway. J Biol Chem. 2001;276(48):45184-45192.

23. Vidal B, et al. Fibrinogen drives dystrophic muscle fibrosis via a TGF $\beta /$ alternative macrophage activation pathway. Genes Dev. 2008;22(13):1747-1752.

24. Wynn TA. Cellular and molecular mechanisms of fibrosis. J Pathol. 2008;214(2):199-210.

25. Wynn TA. Fibrotic disease and the $\mathrm{T}(\mathrm{H}) 1 / \mathrm{T}(\mathrm{H}) 2$ paradigm. Nat Rev Immunol. 2004;4(8):583-594.

26. Pankov R, Yamada KM. Fibronectin at a glance. JCell Sci. 2002;115(pt 20):3861-3863.

27. Amatangelo MD, Bassi DE, Klein-Szanto AJ, Cukierman E. Stroma-derived three-dimensional matrices are necessary and sufficient to promote desmoplastic differentiation of normal fibroblasts. Am J Pathol. 2005;167(2):475-488.

28. Martino MM, Hubbell JA. The 12th-14th type III repeats of fibronectin function as a highly promiscuous growth factor-binding domain. FASEB J. 2010;24(12):4711-4721.

29. Miyamoto S, Teramoto H, Gutkind JS, Yamada KM. Integrins can collaborate with growth factors for phosphorylation of receptor tyrosine kinases and MAP kinase activation: roles of integrin aggregation and occupancy of receptors. JCell Biol. 1996;135(6 pt 1):1633-1642.

30. Clark RA. Synergistic signaling from extracellular matrix-growth factor complexes. J Invest Dermatol. 2008;128(6):1354-1355.

31. Morgan MR, Humphries MJ, Bass MD. Synergistic control of cell adhesion by integrins and syndecans. Nat Rev Mol Cell Biol. 2007;8(12):957-969.
32. Wipff PJ, Rifkin DB, Meister JJ, Hinz B. Myofibroblast contraction activates latent TGF$\beta 1$ from the extracellular matrix. JCell Biol. 2007;179(6):1311-1323.

33. Hinz B. The extracellular matrix and transforming growth factor- $\beta 1$ : tale of a strained relationship. Matrix Biol. 2015;47:54-65.

34. Cao L, et al. Detection of an integrin-binding mechanoswitch within fibronectin during tissue formation and fibrosis. ACS Nano. 2017;11(7):7110-7117.

35. Markowski MC, Brown AC, Barker TH. Directing epithelial to mesenchymal transition through engineered microenvironments displaying orthogonal adhesive and mechanical cues. J Biomed Mater Res A. 2012;100(8):2119-2127.

36. Huang $X$, et al. Matrix stiffness-induced myofibroblast differentiation is mediated by intrinsic mechanotransduction. Am J Respir Cell Mol Biol. 2012;47(3):340-348.

37. Liu F, et al. Feedback amplification of fibrosis through matrix stiffening and COX-2 suppression. JCell Biol. 2010;190(4):693-706.

38. Paszek MJ, et al. Tensional homeostasis and the malignant phenotype. Cancer Cell. 2005;8(3):241-254.

39. Sahai E, Marshall CJ. Differing modes of tumour cell invasion have distinct requirements for Rho/ ROCK signalling and extracellular proteolysis. Nat Cell Biol. 2003;5(8):711-719.

40. Goetz JG, et al. Biomechanical remodeling of the microenvironment by stromal caveolin-1 favors tumor invasion and metastasis. Cell. 2011;146(1):148-163.

41. Li S, et al. Hydrogels with precisely controlled integrin activation dictate vascular patterning and permeability. Nat Mater. 2017;16(9):953-961.

42. Hinz B. Matrix mechanics and regulation of the fibroblast phenotype. Periodontol 2000. 2013;63(1):14-28.

43. Sottile J, Hocking DC. Fibronectin polymerization regulates the composition and stability of extracellular matrix fibrils and cell-matrix adhesions. Mol Biol Cell. 2002;13(10):3546-3559.

44. Quail DF, Joyce JA. Microenvironmental regulation of tumor progression and metastasis. Nat Med. 2013;19(11):1423-1437.

45. Hanahan D, Coussens LM. Accessories to the crime: functions of cells recruited to the tumor microenvironment. Cancer Cell. 2012;21(3):309-322.

46. Pietras K, Ostman A. Hallmarks of cancer: interactions with the tumor stroma. Exp Cell Res. 2010;316(8):1324-1331.

47. Kalluri R. The biology and function of fibroblasts in cancer. Nat Rev Cancer. 2016;16(9):582-598.

48. Chang HY, et al. Diversity, topographic differentiation, and positional memory in 
human fibroblasts. Proc Natl Acad Sci U S A. 2002;99(20):12877-12882.

49. Hematti P. Mesenchymal stromal cells and fibroblasts: a case of mistaken identity? Cytotherapy. 2012;14(5):516-521.

50. Micallef L, Vedrenne N, Billet F, Coulomb B, Darby IA, Desmoulière A. The myofibroblast, multiple origins for major roles in normal and pathological tissue repair. Fibrogenesis Tissue Repair. 2012;5(suppl 1):S5.

51. Rønnov-Jessen L, Petersen OW. Induction of alpha-smooth muscle actin by transforming growth factor-beta 1 in quiescent human breast gland fibroblasts. Implications for myofibroblast generation in breast neoplasia. Lab Invest. 1993;68(6):696-707.

52. Tomasek JJ, Gabbiani G, Hinz B, Chaponnier C, Brown RA. Myofibroblasts and mechanoregulation of connective tissue remodelling. Nat Rev Mol Cell Biol. 2002;3(5):349-363.

53. Rodemann HP, Müller GA. Characterization of human renal fibroblasts in health and disease: II. In vitro growth, differentiation, and collagen synthesis of fibroblasts from kidneys with interstitial fibrosis. Am J Kidney Dis. 1991;17(6):684-686.

54. Simian M, Hirai Y, Navre M, Werb Z, Lochter A, Bissell MJ. The interplay of matrix metalloproteinases, morphogens and growth factors is necessary for branching of mammary epithelial cells. Development. 2001;128(16):3117-3131.

55. Öhlund D, et al. Distinct populations of inflammatory fibroblasts and myofibroblasts in pancreatic cancer. JExp Med. 2017;214(3):579-596.

56. Vaheri A, Enzerink A, Räsänen K, Salmenperä P. Nemosis, a novel way of fibroblast activation, in inflammation and cancer. Exp Cell Res. 2009;315(10):1633-1638.

57. Mishra DK, et al. Human lung fibroblasts inhibit non-small cell lung cancer metastasis in ex vivo 4D model. Ann Thorac Surg. 2015;100(4):11671174; discussion 1174.

58. David CJ, et al. TGF- $\beta$ tumor suppression through a lethal EMT. Cell. 2016;164(5):1015-1030.

59. Li CX, et al. MicroRNA-21 preserves the fibrotic mechanical memory of mesenchymal stem cells. Nat Mater. 2017;16(3):379-389.

60. Liu F, et al. Mechanosignaling through YAP and TAZ drives fibroblast activation and fibrosis. Am J Physiol Lung Cell Mol Physiol. 2015;308(4):L344-L357.

61. Pankova D, et al. Cancer-associated fibroblasts induce a collagen cross-link switch in tumor stroma. Mol Cancer Res. 2016;14(3):287-295.

62. Franco-Barraza J, et al. Matrix-regulated integrin $\alpha v \beta 5$ maintains $\alpha 5 \beta 1$-dependent desmoplastic traits prognostic of neoplastic recurrence. Elife. 2017;6:e20600.

63. Lafyatis R. Transforming growth factor $\beta$ - at the centre of systemic sclerosis. Nat Rev Rheumatol. 2014;10(12):706-719.

64. Alameddine HS, Morgan JE. Matrix metalloproteinases and tissue inhibitor of metalloproteinases in inflammation and fibrosis of skeletal muscles. J Neuromuscul Dis. 2016;3(4):455-473.

65. Yamauchi M, Sricholpech M. Lysine posttranslational modifications of collagen. Essays Biochem. 2012;52:113-133.

66. Myllyharju J, Kivirikko KI. Collagens, modifying enzymes and their mutations in humans, flies and worms. Trends Genet. 2004;20(1):33-43.

67. Bella J, Hulmes DJ. Fibrillar collagens. Subcell Biochem. 2017;82:457-490.

68. Valtavaara M, Papponen H, Pirttilä AM, Hiltunen K, Helander H, Myllylä R. Cloning and characterization of a novel human lysyl hydroxylase isoform highly expressed in pancreas and muscle. J Biol Chem. 1997;272(11):6831-6834.

69. Valtavaara M, Szpirer C, Szpirer J, Myllylä R. Primary structure, tissue distribution, and chromosomal localization of a novel isoform of lysyl hydroxylase (lysyl hydroxylase 3). J Biol Chem. 1998;273(21):12881-12886.

70. Kivirikko KI, Myllylä R, Pihlajaniemi T. Protein hydroxylation: prolyl 4-hydroxylase, an enzyme with four cosubstrates and a multifunctional subunit. FASEB J. 1989;3(5):1609-1617.

71. Schegg B, Hülsmeier AJ, Rutschmann C, Maag C, Hennet T. Core glycosylation of collagen is initiated by two $\beta(1-O)$ galactosyltransferases. $M o l$ Cell Biol. 2009;29(4):943-952.

72. Sricholpech M, Perdivara I, Nagaoka H, Yokoyama M, Tomer KB, Yamauchi M. Lysyl hydroxylase 3 glucosylates galactosylhydroxylysine residues in type I collagen in osteoblast culture. J Biol Chem. 2011;286(11):8846-8856.

73. Sricholpech M, et al. Lysyl hydroxylase 3mediated glucosylation in type I collagen: molecular loci and biological significance. J Biol Chem. 2012;287(27):22998-23009.

74. Terajima M, et al. Glycosylation and crosslinking in bone type I collagen. J Biol Chem. 2014;289(33):22636-22647.

75. Terajima M, et al. Cyclophilin-B modulates collagen cross-linking by differentially affecting lysine hydroxylation in the helical and telopeptidyl domains of tendon type I collagen. J Biol Chem. 2016;291(18):9501-9512.

76. Ishikawa Y, Boudko S, Bächinger HP. Ziploc-ing the structure: triple helix formation is coordinated by rough endoplasmic reticulum resident PPIases. Biochim Biophys Acta. 2015;1850(10):1983-1993.

77. Cabral WA, et al. Abnormal type I collagen post-translational modification and crosslinking in a cyclophilin B KO mouse model of recessive osteogenesis imperfecta. PLoS Genet. 2014;10(6):e1004465.

78. Heard ME, et al. Sc65-null mice provide evidence for a novel endoplasmic reticulum complex regulating collagen lysyl hydroxylation. PLoS Genet. 2016;12(4):e1006002.

79. Gjaltema RA, van der Stoel MM, Boersema M, Bank RA. Disentangling mechanisms involved in collagen pyridinoline cross-linking: the immunophilin FKBP65 is critical for dimerization of lysyl hydroxylase 2. Proc Natl Acad Sci U S A. 2016;113(26):7142-7147.

80. Chen Y, et al. FKBP65-dependent peptidyl-prolyl isomerase activity potentiates the lysyl hydroxylase 2-driven collagen cross-link switch. Sci Rep. 2017;7:46021.

81. Gjaltema RA, Bank RA. Molecular insights into prolyl and lysyl hydroxylation of fibrillar collagens in health and disease. Crit Rev Biochem Mol Biol. 2017;52(1):74-95.

82. Duran I, et al. A chaperone complex formed by HSP47, FKBP65, and BiP modulates telopeptide lysyl hydroxylation of type I procollagen. J Bone Miner Res. 2017;32(6):1309-1319.

83. Eyre DR, Weis MA. Bone collagen: new clues to its mineralization mechanism from recessive osteogenesis imperfecta. Calcif Tissue Int. 2013;93(4):338-347.

84. Kang H, Aryal ACS, Marini JC. Osteogenesis imperfecta: new genes reveal novel mechanisms in bone dysplasia. Transl Res. 2017;181:27-48.

85. Csiszar K. Lysyl oxidases: a novel multifunctional amine oxidase family. Prog Nucleic Acid Res Mol Biol. 2001;70:1-32.

86. Kagan HM, Li W. Lysyl oxidase: properties, specificity, and biological roles inside and outside of the cell. J Cell Biochem. 2003;88(4):660-672.

87. Trackman PC. Enzymatic and non-enzymatic functions of the lysyl oxidase family in bone. Matrix Biol. 2016;52-54:7-18.

88. Yamauchi M, Mechanic GL. Cross-linking of collagen. In: Nimni ME, ed. Collagen. Boca Raton, Florida, USA: CRC Press; 1988:157-172.

89. Yamauchi M, Chandler GS, Tanzawa H, Katz EP. Cross-linking and the molecular packing of corneal collagen. Biochem Biophys Res Commun. 1996;219(2):311-315.

90. Tanzer ML, Housley T, Berube L, Fairweather R, Franzblau C, Gallop PM. Structure of two histidine-containing crosslinks from collagen. J Biol Chem. 1973;248(2):393-402.

91. Bernstein PH, Mechanic GL. A natural histidine-based imminium cross-link in collagen and its location. J Biol Chem. 1980;255(21):10414-10422.

92. Bailey AJ, Paul RG, Knott L. Mechanisms of maturation and ageing of collagen. Mech Ageing Dev. 1998;106(1-2):1-56.

93. Kirschmann DA, Seftor EA, Nieva DR, Mariano EA, Hendrix MJ. Differentially expressed genes associated with the metastatic phenotype in breast cancer. Breast Cancer Res Treat. 1999;55(2):127-136

94. Akiri G, et al. Lysyl oxidase-related protein-1 promotes tumor fibrosis and tumor progression in vivo. Cancer Res. 2003;63(7):1657-1666.

95. Barry-Hamilton V, et al. Allosteric inhibition of lysyl oxidase-like-2 impedes the development of a pathologic microenvironment. Nat Med. 2010;16(9):1009-1017.

96. Cox TR, et al. LOX-mediated collagen crosslinking is responsible for fibrosis-enhanced metastasis. Cancer Res. 2013;73(6):1721-1732.

97. Barker HE, Cox TR, Erler JT. The rationale for targeting the LOX family in cancer. Nat Rev Cancer. 2012;12(8):540-552.

98. Park JS, Lee JH, Lee YS, Kim JK, Dong SM, Yoon DS. Emerging role of LOXL2 in the promotion of pancreas cancer metastasis. Oncotarget. 2016;7(27):42539-42552.

99. Martin A, et al. Lysyl oxidase-like 2 represses Notch1 expression in the skin to promote squamous cell carcinoma progression. ЕМВО J. 2015;34(8):1090-1109.

100.Wong CC, et al. Hypoxia-inducible factor 1 is a master regulator of breast cancer metastatic niche formation. Proc Natl Acad Sci U S A. 2011;108(39):16369-16374

101.Levental KR, et al. Matrix crosslinking forces tumor progression by enhancing integrin signal- 
ing. Cell. 2009;139(5):891-906.

102. Brinckmann J, et al. Different pattern of collagen cross-links in two sclerotic skin diseases: lipodermatosclerosis and circumscribed scleroderma. J Invest Dermatol. 2001;117(2):269-273.

103. Uzawa K, Marshall MK, Katz EP, Tanzawa H, Yeowell HN, Yamauchi M. Altered posttranslational modifications of collagen in keloid. Biochem Biophys Res Commun. 1998;249(3):652-655.

104.Last JA, King TE, Nerlich AG, Reiser KM. Collagen cross-linking in adult patients with acute and chronic fibrotic lung disease. Molecular markers for fibrotic collagen. Am Rev Respir Dis. 1990;141(2):307-313.

105. Gerriets JE, Reiser KM, Last JA. Lung collagen cross-links in rats with experimentally induced pulmonary fibrosis. Biochim Biophys Acta. 1996;1316(2):121-131.

106. Ricard-Blum S, Liance M, Houin R, Grimaud JA, Vuitton DA. Covalent cross-linking of liver collagen by pyridinoline increases in the course of experimental alveolar echinococcosis. Parasite. 1995;2(2):113-118.

107. Brenner DA, et al. New aspects of hepatic fibrosis. J Hepatol. 2000;32(1 Suppl):32-38.

108. Perepelyuk M, et al. Hepatic stellate cells and portal fibroblasts are the major cellular sources of collagens and lysyl oxidases in normal liver and early after injury. Am J Physiol Gastrointest Liver Physiol. 2013;304(6):G605-G614.

109. van der Slot AJ, et al. Identification of PLOD2 as telopeptide lysyl hydroxylase, an important enzyme in fibrosis. J Biol Chem. 2003;278(42):40967-40972.

110.van der Slot AJ, et al. Increased formation of pyridinoline cross-links due to higher telopeptide lysyl hydroxylase levels is a general fibrotic phenomenon. Matrix Biol. 2004;23(4):251-257.

111. Xu Y, et al. Procollagen-lysine 2-oxoglutarate 5 -dioxygenase 2 promotes hypoxia-induced glioma migration and invasion. Oncotarget. 2017;8(14):23401-23413.

112. Gilkes DM, et al. Procollagen lysyl hydroxylase 2 is essential for hypoxia-induced breast cancer metastasis. Mol Cancer Res. 2013;11(5):456-466.

113. Eisinger-Mathason TS, et al. Hypoxia-dependent modification of collagen networks promotes sarcoma metastasis. Cancer Discov. 2013;3(10):1190-1205.

114. Noda T, et al. PLOD2 induced under hypoxia is a novel prognostic factor for hepatocellular carcinoma after curative resection. Liver Int. 2012;32(1):110-118.

115. Chen Y, et al. Lysyl hydroxylase 2 induces a collagen cross-link switch in tumor stroma. JClin Invest. 2015;125(3):1147-1162.

116. Sada M, et al. Hypoxic stellate cells of pancreatic cancer stroma regulate extracellular matrix fiber organization and cancer cell motility. Cancer Lett. 2016;372(2):210-218.

117. Wang M, et al. HIF-1 $\alpha$ promoted vasculogenic mimicry formation in hepatocellular carcinoma through LOXL2 up-regulation in hypoxic tumor microenvironment. J Exp Clin Cancer Res. 2017;36(1):60.

118. Kasashima H, et al. Lysyl oxidase is associated with the epithelial-mesenchymal transition of gastric cancer cells in hypoxia. Gastric Cancer.
2016;19(2):431-442.

119. Miller BW, et al. Targeting the LOX/hypoxia axis reverses many of the features that make pancreatic cancer deadly: inhibition of LOX abrogates metastasis and enhances drug efficacy. EMBO Mol Med. 2015;7(8):1063-1076.

120. Ji F, et al. Hypoxia inducible factor $1 \alpha$-mediated LOX expression correlates with migration and invasion in epithelial ovarian cancer. Int JOncol. 2013;42(5):1578-1588.

121. Erler JT, et al. Hypoxia-induced lysyl oxidase is a critical mediator of bone marrow cell recruitment to form the premetastatic niche. Cancer Cell. 2009;15(1):35-44.

122. Chang J, Erler J. Hypoxia-mediated metastasis. Adv Exp Med Biol. 2014;772:55-81.

123. Conklin MW, et al. Aligned collagen is a prognostic signature for survival in human breast carcinoma. Am J Pathol. 2011;178(3):1221-1232.

124. Myllylä R, et al. Expanding the lysyl hydroxylase toolbox: new insights into the localization and activities of lysyl hydroxylase 3 (LH3). JCell Physiol. 2007;212(2):323-329.

125. Gascard P, Tlsty TD. Carcinoma-associated fibroblasts: orchestrating the composition of malignancy. Genes Dev. 2016;30(9):1002-1019.

126.Chen Y, et al. Lysyl hydroxylase 2 is secreted by tumor cells and can modify collagen in the extracellular space. J Biol Chem. 2016;291(50):25799-25808.

127. Sharma P, Allison JP. Immune checkpoint targeting in cancer therapy: toward combination strategies with curative potential. Cell. 2015;161(2):205-214.

128. Peterson LW, Artis D. Intestinal epithelial cells: regulators of barrier function and immune homeostasis. Nat Rev Immunol. 2014;14(3):141-153.

129. Nowarski R, Jackson R, Flavell RA. The stromal intervention: regulation of immunity and inflammation at the epithelial-mesenchymal barrier. Cell. 2017;168(3):362-375.

130. Hallmann R, et al. The regulation of immune cell trafficking by the extracellular matrix. Curr Opin Cell Biol. 2015;36:54-61.

131. Mouw JK, Ou G, Weaver VM. Extracellular matrix assembly: a multiscale deconstruction. Nat Rev Mol Cell Biol. 2014;15(12):771-785.

132. Lämmermann $\mathrm{T}$, et al. Rapid leukocyte migration by integrin-independent flowing and squeezing. Nature. 2008;453(7191):51-55.

133. Wolf K, Müller R, Borgmann S, Bröcker EB, Friedl P. Amoeboid shape change and contact guidance: T-lymphocyte crawling through fibrillar collagen is independent of matrix remodeling by MMPs and other proteases. Blood. 2003;102(9):3262-3269.

134. Bruckner P. Suprastructures of extracellular matrices: paradigms of functions controlled by aggregates rather than molecules. Cell Tissue Res. 2010;339(1):7-18.

135. Riopel MM, Li J, Liu S, Leask A, Wang R. $\beta 1$ Integrin-extracellular matrix interactions are essential for maintaining exocrine pancreas architecture and function. Lab Invest. 2013;93(1):31-40.

136. Wu C, et al. Endothelial basement membrane laminin alpha5 selectively inhibits $\mathrm{T}$ lymphocyte extravasation into the brain. Nat Med. 2009;15(5):519-527.
137. Kenne E, Soehnlein O, Genové G, Rotzius P, Eriksson EE, Lindbom L. Immune cell recruitment to inflammatory loci is impaired in mice deficient in basement membrane protein laminin alpha4. J Leukoc Biol. 2010;88(3):523-528.

138. Alexander J, Cukierman E. Stromal dynamic reciprocity in cancer: intricacies of fibroblastic-ECM interactions. Curr Opin Cell Biol. 2016;42:80-93.

139. Costea DE, et al. Identification of two distinct carcinoma-associated fibroblast subtypes with differential tumor-promoting abilities in oral squamous cell carcinoma. Cancer Res. 2013;73(13):3888-3901.

140. De Boeck A, et al. Differential secretome analysis of cancer-associated fibroblasts and bone marrowderived precursors to identify microenvironmental regulators of colon cancer progression. Proteomics. 2013;13(2):379-388.

141. Lotti F, et al. Chemotherapy activates cancerassociated fibroblasts to maintain colorectal cancer-initiating cells by IL-17A. J Exp Med. 2013;210(13):2851-2872.

142. Erez N, Truitt M, Olson P, Arron ST, Hanahan D. Cancer-associated fibroblasts are activated in incipient neoplasia to orchestrate tumorpromoting inflammation in an NF- $\mathrm{BB}$-dependent manner. Cancer Cell. 2010;17(2):135-147.

143. Feig C, et al. Targeting CXCL12 from FAPexpressing carcinoma-associated fibroblasts synergizes with anti-PD-L1 immunotherapy in pancreatic cancer. Proc Natl Acad Sci U S A. 2013;110(50):20212-20217.

144.Ene-Obong A, et al. Activated pancreatic stellate cells sequester $\mathrm{CD} 8^{+} \mathrm{T}$ cells to reduce their infiltration of the juxtatumoral compartment of pancreatic ductal adenocarcinoma. Gastroenterology. 2013;145(5):1121-1132.

145. Chang $\mathrm{CH}$, et al. Metabolic competition in the tumor microenvironment is a driver of cancer progression. Cell. 2015;162(6):1229-1241.

146.Su X, Ye J, Hsueh EC, Zhang Y, Hoft DF, Peng G. Tumor microenvironments direct the recruitment and expansion of human Th17 cells. JImmunol. 2010;184(3):1630-1641.

147. Barnas JL, Simpson-Abelson MR, Brooks SP, Kelleher RJ, Bankert RB. Reciprocal functional modulation of the activation of $\mathrm{T}$ lymphocytes and fibroblasts derived from human solid tumors. J Immunol. 2010;185(5):2681-2692.

148. Chen W, et al. Conversion of peripheral $\mathrm{CD} 4{ }^{+} \mathrm{CD} 25^{-}$naive $\mathrm{T}$ cells to $\mathrm{CD} 4^{+} \mathrm{CD} 25^{+}$regulatory $\mathrm{T}$ cells by TGF- $\beta$ induction of transcription factor Foxp3. J Exp Med. 2003;198(12):1875-1886.

149. De Monte L, et al. Intratumor T helper type 2 cell infiltrate correlates with cancer-associated fibroblast thymic stromal lymphopoietin production and reduced survival in pancreatic cancer. J Exp Med. 2011;208(3):469-478.

150. Schaefer L. Complexity of danger: the diverse nature of damage-associated molecular patterns. J Biol Chem. 2014;289(51):35237-35245.

151. Schaefer L, Tredup C, Gubbiotti MA, Iozzo RV. Proteoglycan neofunctions: regulation of inflammation and autophagy in cancer biology. FEBS J. 2017;284(1):10-26.

152. Salmon H, et al. Matrix architecture defines the preferential localization and migration of $\mathrm{T}$ cells into the stroma of human lung tumors. J Clin 
Invest. 2012;122(3):899-910.

153. Hartmann N, et al. Prevailing role of contact guidance in intrastromal T-cell trapping in human pancreatic cancer. Clin Cancer Res. 2014;20(13):3422-3433.

154. Wolf K, et al. Physical limits of cell migration: control by ECM space and nuclear deformation and tuning by proteolysis and traction force. JCell Biol. 2013;201(7):1069-1084.

155. Carstens JL, et al. Spatial computation of intratumoral $\mathrm{T}$ cells correlates with survival of patients with pancreatic cancer. Nat Commun. 2017;8:15095.

156. Smigiel KS, Parks WC. Matrix metalloproteinases and leukocyte activation. Prog Mol Biol Transl Sci. 2017;147:167-195.

157. Liu YM, Nepali K, Liou JP. Idiopathic pulmonary fibrosis: current status, recent progress, and emerging targets. JMed Chem. 2017;60(2):527-553.

158. Di Sario A, et al. Effect of pirfenidone on rat hepatic stellate cell proliferation and collagen production. J Hepatol. 2002;37(5):584-591.

159. Hewitson TD, et al. Pirfenidone reduces in vitro rat renal fibroblast activation and mitogenesis. J Nephrol. 2001;14(6):453-460.

160. Ozes ON, Blatt LM. Development of a high throughput collagen assay using a cellular model of idiopathic pulmonary fibrosis. Chest. 2006;130:230S.

161. Grattendick KJ, Nakashima JM, Feng L, Giri SN, Margolin SB. Effects of three anti-TNF- $\alpha$ drugs: etanercept, infliximab and pirfenidone on release of TNF- $\alpha$ in medium and TNF- $\alpha$ associated with the cell in vitro. Int Immunopharmacol. 2008;8(5):679-687.

162. Schaefer CJ, Ruhrmund DW, Pan L, Seiwert SD, Kossen K. Antifibrotic activities of pirfenidone in animal models. Eur Respir Rev. 2011;20(120):85-97.

163. Selman M, King TE, Pardo A, American Thoracic Society, European Respiratory Society, American College of Chest Physicians. Idiopathic pulmonary fibrosis: prevailing and evolving hypotheses about its pathogenesis and implications for therapy. Ann Intern Med. 2001;134(2):136-151.
164. Wollin L, Maillet I, Quesniaux V, Holweg A, Ryffel B. Antifibrotic and anti-inflammatory activity of the tyrosine kinase inhibitor nintedanib in experimental models of lung fibrosis. J Pharmacol Exp Ther. 2014;349(2):209-220.

165. Wang J, Chen J, Guo Y, Wang B, Chu H. Strategies targeting angiogenesis in advanced non-small cell lung cancer. Oncotarget. 2017;8(32):53854-53872.

166. Henderson NC, et al. Targeting of $\alpha \mathrm{v}$ integrin identifies a core molecular pathway that regulates fibrosis in several organs. Nat Med. 2013;19(12):1617-1624.

167. Stylianopoulos T. The solid mechanics of cancer and strategies for improved therapy. J Biomech Eng. 2017;139(2):10.1115/1.4034991.

168. Polydorou C, Mpekris F, Papageorgis P, Voutouri $\mathrm{C}$, Stylianopoulos T. Pirfenidone normalizes the tumor microenvironment to improve chemotherapy. Oncotarget. 2017;8(15):24506-24517.

169. Mpekris F, et al. Sonic-hedgehog pathway inhibition normalizes desmoplastic tumor microenvironment to improve chemo- and nanotherapy. J Control Release. 2017;261:105-112.

170.Chang J, et al. Pre-clinical evaluation of small molecule LOXL2 inhibitors in breast cancer. Oncotarget. 2017;8(16):26066-26078.

171. Cox TR, et al. The hypoxic cancer secretome induces pre-metastatic bone lesions through lysyl oxidase. Nature. 2015;522(7554):106-110.

172. Baker AM, Bird D, Lang G, Cox TR, Erler JT. Lysyl oxidase enzymatic function increases stiffness to drive colorectal cancer progression through FAK. Oncogene. 2013;32(14):1863-1868.

173. Baker AM, et al. Lysyl oxidase plays a critical role in endothelial cell stimulation to drive tumor angiogenesis. Cancer Res. 2013;73(2):583-594.

174. Baker AM, et al. The role of lysyl oxidase in SRC-dependent proliferation and metastasis of colorectal cancer. J Natl Cancer Inst. 2011;103(5):407-424.

175. Meissner EG, et al. Simtuzumab treatment of advanced liver fibrosis in HIV and HCV-infected adults: results of a 6-month open-label safety trial. Liver Int. 2016;36(12):1783-1792.

176. Miller BW, et al. Targeting the LOX/hypoxia axis reverses many of the features that make pancreatic cancer deadly: inhibition of LOX abrogates metastasis and enhances drug efficacy. $E M B O$ Mol Med.2015;7(8):1063-1076.

177. Benson $\mathrm{AB}$, et al. A phase II randomized, doubleblind, placebo-controlled study of simtuzumab or placebo in combination with gemcitabine for the first-line treatment of pancreatic adenocarcinoma. Oncologist. 2017;22(3):241-e15.

178. Hecht JR, Benson AB, Vyushkov D, Yang Y, Bendell J, Verma U. A phase II, randomized, double-blind, placebo-controlled study of simtuzumab in combination with FOLFIRI for the second-line treatment of metastatic KRAS mutant colorectal adenocarcinoma. Oncologist. 2017;22(3):243-e23.

179. Rhim AD, et al. EMT and dissemination precede pancreatic tumor formation. Cell.2012; 148(1-2):349-361.

180. Du H, Pang M, Hou X, Yuan S, Sun L. PLOD2 in cancer research. Biomed Pharmacother. 2017;90:670-676.

181. Olive KP, et al. Inhibition of Hedgehog signaling enhances delivery of chemotherapy in a mouse model of pancreatic cancer. Science. 2009;324(5933):1457-1461.

182. Rhim AD, et al. Stromal elements act to restrain, rather than support, pancreatic ductal adenocarcinoma. Cancer Cell. 2014;25(6):735-747.

183. Özdemir BC, et al. Depletion of carcinomaassociated fibroblasts and fibrosis induces immunosuppression and accelerates pancreas cancer with reduced survival. Cancer Cell. 2014;25(6):719-734.

184. Sherman $\mathrm{MH}$, et al. Vitamin D receptor-mediated stromal reprogramming suppresses pancreatitis and enhances pancreatic cancer therapy. Cell. 2014;159(1):80-93.

185. Beatty GL, et al. CD40 agonists alter tumor stroma and show efficacy against pancreatic carcinoma in mice and humans. Science. 2011;331(6024):1612-1616. 